\title{
PMOE planetary/lunar ephemeris framework $\dagger$
}

\author{
G. Y. Li ${ }^{1}$, H. B. Zhao ${ }^{1,2}$, Y. Xia ${ }^{1,2}$, F. Zeng ${ }^{1,2}$ and Y. J. Luo ${ }^{1,2}$ \\ ${ }^{1}$ Purple Mountain Observatory, Chinese Academy of Sciences, Nanjing 210008, China \\ email: gyl@pmo.ac.cn \\ ${ }^{2}$ Graduate University of Chinese Academy of Sciences, Beijing 100049, China
}

\begin{abstract}
The PMOE planetary/lunar ephemeris framework was established in 2003, and has been improved in recent years. In the framework of the post-Newtonian effects, the figure perturbation effects arising from the a finite size of the Sun, Moon and the Earth, and the effect of the Earth tide were taken into account. The accuracy of using the PMOE ephemeris to predict the positions of the planets in the solar system are the same as that of JPL DE 405. Based on this framework, the orbit optimization for the LISA, ASTROD and ASTROD I missions, and the computation of celestial phenomena and lunar phases in the Xia Shang and Zhou period of ancient China have been completed.
\end{abstract}

Keywords. astrometry, ephemerides, methods: data analysis, methods: numerical

\section{Introduction}

E.M. Standish and J.G. Williams et al. in the NASA JPL published the DE/LE planetary/lunar ephemerides based on observations of the radar ranging, lunar laser raging (LLR) and others in the solar system after 1960s. The important DE/LE ephemerides are DE/LE 96 in 1975, DE/LE 102 in 1977 (Newhall, Standish \& Williams 1983), DE/LE 200 in 1982 (Standish 1990), DE/LE 403 in 1995, and DE/LE 405 in 1998 (Standish 1998). Based upon the resolutions of IAU, DE/LE 200 and DE/LE 405 were acknowledged generally as the standards of the ephemerides. P. Bretagnon and G. Francou et al. in the IMCCE and Bureau International de l'Heure published the VSOP 87 (Bretagnon \& Francou1988), VSOP 2000 (Bretagnon, 2001), PS-1996 solar/planetary ephemerides and ELP 2000 lunar ephemeris (Chapront-Touz \& J. Chapront, 1983) based on the DE 200 and DE 403 ephemerides. G.A. Krasinsky and E.V. Pitjeva at the Institute of Applied Astronomy of RAS published the EPM 1998, EPM 2000 and EPM 2002 ephemerides (Pitjeva, 2001).

In China, Jiaxiang Zhang studied the orbital evalution of the comets $60 \mathrm{P} /$ Tsuchinshan 2, 62P/Tsuchinshan 1, 1P/Halley and asteroid Icarus with his model and improved Cowell integrator in 1970s. Zhang made a series of successful predictions of the impact of Comet Shoemaker-Levy 9 on Jupiter in 1994 which compared with Galileo's observation yielded the RMS of 8'.46 (Zhang 1996). The predictions given by Zhang and those of JPL are about the same accuracy. Using the same ephemeris, Guangyu Li and Haibin Zhao found the origin of particles in the second peak of the Leonids in 1998 ( $\mathrm{Li} \&$ Zhao 2002). At the same time Weitou Ni et al. from the Center for Gravitation and Cosmology of Tsing Hua University published the CGC 1 and CGC 2 ephemeris frame, and worked on the orbit simulation and the parameter determination for the ASTROD and ASTROD I mission concepts (Ni 2006).

$\dagger$ Supported by Foundation of Minor Planets of Purple Mountain Observatory and the National Science Foundation (Grant No. 10503013) 
According to the requirement of the solar system exploration, especially for the study of the ASTROD I mission, in 2001 Guangyu Li cooperated with Weitou Ni to build a more accurate ephemeris. By the end of the 2002 the PMOE 2003 ephemeris framework had been published ( $\mathrm{Li} 2003$ ) and was further improved in 2005.

\section{PMOE ephemeris framework}

Referred to the Zhang's Model and CGC 2, the dynamical model of the solar system and the integrator of the PMOE 2003 ephemeris framework was proposed. The following effects on the bodies in the solar system have been taken into consideration: (1)the postNewtonian effects; (2) the figure perturbation effects arising from the finite size of the Sun, the Moon and the Earth; (3) the effect of the Earth tide on the motion of the Moon; (4) effects of gravitational forces of the Big 3 and 314 other large asteroids. With the identical astronomical constants, initial position and velocities as those in DE 405 ephemeris, the orbits of the Earth-Moon barycenter, 7 planets, Pluto and the Moon around the Earth, are integrated for 1,200 days and 36,000 days (about 98 years), the differences between PMOE and DE 405 ephemeris are shown in Table 1, Table 2 and Figure 1, respectively.

Table 1. Differences between PMOE and DE 405 after integrating 1,200 days

\begin{tabular}{cccc}
\hline Bodies & $\begin{array}{c}\text { Heliocentric distance } \\
\Delta \mathrm{r}(\mathrm{m})\end{array}$ & $\begin{array}{c}\text { Heliocentric longitude } \\
\Delta \lambda(\mathrm{mas})\end{array}$ & $\begin{array}{c}\text { Heliocentric latitude } \\
\delta(\mathrm{mas})\end{array}$ \\
\hline Mercury & $-3.1-3.1$ & $-0.3-0.2$ & $-0.0-0.01$ \\
Venus & $-9.9-9.6$ & $-0.03-0.04$ & $-0.0-0.02$ \\
Earth & $-3.3-2.9$ & $0.0-0.04$ & $-0.1-0.0$ \\
Moon* & $-0.13-0.13$ & $-1.4-0.0$ & $-0.6-0.5$ \\
Earth-Moon & $-3.3-2.8$ & $0.0-0.04$ & $-0.1-0.01$ \\
Mars & $-25.6-14.7$ & $-0.02-0.08$ & $-0.01-0.03$ \\
Jupiter & $-512.2-0.0$ & $0.0-0.19$ & $0.0-0.02$ \\
Saturn & $-178.6-0.0$ & $0.0-0.02$ & $0.0-0.002$ \\
Uranus & $-62.8-0.0$ & $<0.0004$ & $<0.0002$ \\
Neptune & $-30.1-0.0$ & $<0.0004$ & $<-0.00007$ \\
Pluto & $-26.8-0.0$ & $<-0.0002$ & $<0.0002$ \\
\hline
\end{tabular}

$*$ Geocentric distance, longitude and latitude for the Moon.

Table 2. Differences between PMOE and DE 405 after integrating 36,000 days

\begin{tabular}{cccc}
\hline Bodies & $\begin{array}{c}\text { Heliocentric distance } \\
\Delta \mathrm{r}(\mathrm{m})\end{array}$ & $\begin{array}{c}\text { Heliocentric longitude } \\
\Delta \lambda(\mathrm{mas})\end{array}$ & $\begin{array}{c}\text { Heliocentric latitude } \\
\delta(\mathrm{mas})\end{array}$ \\
\hline Mercury & $-67.6-69.1$ & $-1.6-0.1$ & $-0.5-0.4$ \\
Venus & $-20.9-20.5$ & $-0.3-0.0$ & $-0.1-0.1$ \\
Earth & $-26.5-19.6$ & $0-0.7$ & $-0.2-0.2$ \\
Moon & $-32.2-32.3$ & $-336.3-0.0$ & $-121.3-138.2$ \\
Earth-Moon & $-23.6-17.7$ & $0.0-0.7$ & $-0.2-0.2$ \\
Mars & $-1302-1347$ & $0-10.2$ & $-2.5-3.9$ \\
Jupiter & $-3765-2274$ & $0-17.4$ & $-5.6-6.3$ \\
Saturn & $-1637-419$ & $0-5.0$ & $-1.7-1.6$ \\
Uranus & $-3797-549$ & $0-1.7$ & $-0.7-0.3$ \\
Neptune & $-5023-0$ & $0-1.1$ & $0.0-0.2$ \\
Pluto & $-5628-0$ & $0-0.5$ & $0.0-0.2$ \\
\hline
\end{tabular}

$*$ Geocentric distance, longitude and latitude for the Moon. 

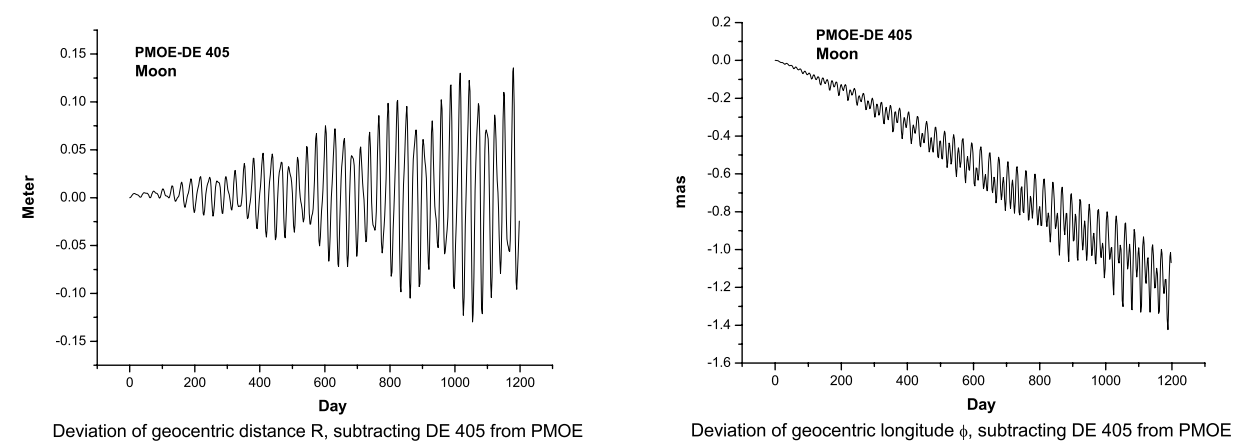

Figure 1. Differences between PMOE and DE 405.

\section{Preliminary applications}

Based on the PMOE 2003 ephemeris framework, Li et al. have calculated the orbits of the LISA, ASTROD I and ASTROD spacecrafts (Ni 2006), and proposed the methods for the orbital optimization of the LISA spacecrafts, cooperating with H. Gerhard et al. at ESA (Li, Yi \& Heinzel 2008). Also the celestial phenomena and lunar phases in Xia Shang and Zhou Period in China (BC 2100-BC 771) have been calculated and published. After further improvements of the lunar dynamical model and fitting the latest ranging data, lander ranging data, VLBI data, and LLR data, an independent Chinese ephemeris will be completed.

Along with the development of the exploration of the solar system and laser ranging between the bodies in the solar system, the precision of the measurements of the bodies in the solar system will be greatly improved. The present planetary/lunar ephemerides based on the first post-Newtonian (1PN) theory, including the DE ephemerides, will not satisfy the requirement of the space exploration. The planetary/lunar ephemeris should be to establish on the basis of the second post-Newtonian (2PN) theory, which is studied by Weitou Ni's group at the Purple Mountain Observatory (Ni 2006).

\section{References}

Bretagnon, P. \& Francou, G. 1988, A\&EA, 202, 309.

Bretagnon, P. 2001, Celest. Mech. \&5 Dyn. Ast., 80, 3, 205.

Chapront-Touzé, M. \& Chapront, J. 1983, A\& $A$ A, 124, 50.

Li, G. \& Zhao, H. 2002, Int. J. Mod. Phys. D, 11, 7, 1021.

Li, G. \& Ni, W. T. 2003, Publications of Purple Mountain Observatory, 22, 3-4, in Chinese .

Li, G., Yi, Z., Heinzel, G., et al., 2008, Int. J. Mod. Phys. D, 17, 6.

Newhall, X. X., Standish, Jr., E. M., \& Williams, J. G. 1983, A\&\&A, 125, 150.

Ni, W. T., et al., 2006, Gen. Rel. Grav., 39.

Pitjeva, E. V. 2001, A\&GA, 371, 760.

Standish, Jr., E.M. 1990, A\&BA, 233, 252.

Standish, Jr., E. M. 1998, JPL IOM 312, F-98-048.

Zhang, J. X., et al., 1996, Science in China (Series A), 39, 2, 207. 